\title{
Restoration of gait by functional electrical stimulation in paraplegic patients: a modified programme of treatment
}

\author{
M Maležič and S Hesse \\ Klinik Berlin, Department of Neurological Rehabilitation, Free University Berlin, Kladower Damm 223, 14089 \\ Berlin, Germany
}

\begin{abstract}
Restoration of standing and of gait by functional electrical stimulation in clinically complete paraplegic patients was modified in the course of treatment and in the stimulation parameters. By substituting an initial cyclic muscle strengthening with an active stimulated standing, four patients with T3-11 lesions started walking with electrical stimulation in 10-17 days. They walked without ankle-foot orthoses. With a satisfactory stride length of $0.75-0.97 \mathrm{~m}$, their gait velocity ranged from very slow to that of a leisurely healthy gait. Already established stimulation of the quadriceps muscles for standing and of the peroneal nerves for lower limb flexion during the swing phase of gait was applied. Diminished limb flexion after several weeks was restored by an increase of the stimulation frequency of the peroneal nerve from 20 to $60 \mathrm{~Hz}$. EMG and kinesiological measurements displayed an improved direct response of the ankle as well as of the reflex mediated hip, knee and ankle flexion response. At the same time stimulation frequency was reduced to $16 \mathrm{~Hz}$ for the quadriceps muscles in order to reduce fatigue.
\end{abstract}

Keywords: functional electrical stimulation (FES); spinal cord injury; standing; gait; flexion response

\section{Introduction}

Clinically complete spinal cord injury patients with a central lesion can regain their standing and walking ability by functional electrical stimulation (FES) of their lower limbs. ${ }^{1}$ A four channel surface electrical stimulation method includes both quadriceps muscles to provide standing, while stepping is induced by the stimulation of combined ankle dorsal flexion and flexion withdrawal response of the peroneal nerve. Alternating stimulation between the quadriceps muscle and peroneal nerve of both lower limbs is controlled with manual switches by the patients themselves. The stimulation frequency around $20 \mathrm{~Hz}$ has been regarded as optimal for both stimulation sites in all phases of the treatment.

Currently used stimulation protocols have included an initial strengthening programme of the antigravity muscles. The patients have been treated by cyclic stimulation of the quadriceps muscles while lying or sitting before the electrically induced standing. The strengthening might have lasted weeks or even months before initiation of the stimulated gait. ${ }^{1}$ The average re-strengthening time was found to be approximately 1 month. ${ }^{2}$ In incomplete spinal cord injury patients, the isotonic strengthening was replaced by a progressive resisted quadriceps conditioning by the stimulation and weights attached at the ankle and lasted 6 months. ${ }^{3}$

On the other hand, it was demonstrated in chronic non-ambulatory hemiparetic patients that an immediate walking with FES without previous strengthening of the muscles could provide gait without the support of an accompanying therapist in 2-3 weeks. ${ }^{4,5}$ Therefore immediate standing assisted by FES without previous conditioning of the muscles was chosen for paraplegic patients in this study.

Stimulation of the flexion reflex during the swing phase may be hindered by habituation after prolonged use. ${ }^{6}$ It could reduce the amount of hip flexion to zero after a number of stimuli. ${ }^{7}$ To improve the limb flexion during the FES gait, single high intensity pulses were added and two stimulation sites were multiplexed. ${ }^{8,9}$ However, the stimulation frequency around $20 \mathrm{~Hz}$ remained in the clinical reports not only for the quadriceps muscles but also for the flexion response.

As relatively high frequencies had been commonly used in basic neurophysiological studies of the flexion reflex in spinal man, ${ }^{10-12}$ effects of stimulation frequencies above $20 \mathrm{~Hz}$ on the FES flexion response were investigated in this study. A presented modified method approached the time course of treatment and the stimulation parameters in order to substitute the preparatory period and to improve the response to stimulation.

\section{Methods}

Four male paraplegic patients aged from 24 to 38 years have so far been treated. They all had clinically complete lesions at T3, T4, T6 and T11 spinal cord levels, resulting from car accidents dating back 16 months to 16 years. Two were inpatients and had two 
FES sessions a day, whilst the other two were outpatients and came for once daily sessions during the first month of treatment. The patients had practised passive standing attached to a standing board; two of them had also used knee-ankle-foot orthoses before the begining of the FES programme.

Two commercially available ALT-2 dual channel stimulators (Jožef Stephan Institute, Ljublyana, Slovenia) were used for the alternating stimulation of the quadriceps muscle and the peroneal nerve on both sides. The stimulators with biphasic constant current pulses up to $150 \mathrm{~mA}$ had a pre-set $20 \mathrm{~Hz}$ frequency and $0.25 \mathrm{~ms}$ pulse duration. Bipolar $8 \times 5 \mathrm{~cm}$ felt pad electrodes were used for the quadriceps muscle, while the peroneal nerve was stimulated by a $2.5 \mathrm{~cm}$ round active electrode, using the distal quadriceps electrode as a neutral one. No additional bracing, eg ankle-foot orthoses, was used besides the FES.

The stimulation programme excluded any former cyclic stimulation in a lying or sitting position. Instead, the patients started to stand by bilateral quadriceps muscle stimulation from the onset. Possible hazards due to disused bone demineralisation in paraplegic patients were reduced by the fact that they had performed passive standing and even braced walking before the begining of the stimulation programme. The patients first used a standing frame with handles and elbow support and switched to a rolling walker before starting electrically stimulated stepping. One of the patients combined the walker with elbow crutches during the later stage of rehabilitation.

With a well established pattern of electrically stimulated gait an Infotronic CDG system of force shoes (Infotronic, Tubbergen, Sweden) and electronic storage of measured data were used for its assessment. The system, additionally calibrated by the slowest possible gait of six healthy volunteers and their measured body weight, provided vertical ground reaction forces, trajectories of their point of action on the feet, stride phase durations, cadence and, with the manually measured distance, stride length and velocity of gait.

With decrease of the combined flexor response in the ankle, knee and hip joints after a couple of months, the stimulation and amplitude had to be subsequently increased in three patients. Besides improved hip and knee flexions at the higher stimulation amplitude, plantar flexion prevailed at the ankle joint, diminishing functionality of the flexor response of the whole lower limb. In two patients the $2.5 \mathrm{~cm}$ peroneal electrodes were replaced by $1 \mathrm{~cm}$ round ones in order to stimulate the nerve by lower amplitudes more selectively. As this was not efficient after several weeks, higher stimulation frequencies were investigated for the elicitation of the flexor response in all patients. The stimulators were modified to $60 \mathrm{~Hz}$ frequency and $0.15 \mathrm{~ms}$ pulse duration of the flexor response channel. The frequency of the quadriceps channel was lowered to $16 \mathrm{~Hz}$ at the same time.

The Medelec Mystro MS 92 electrophysiological system (Medelec Ltd, Surrey, UK) was used for the investigation of the flexor response. Surface EMG signals of the tibialis anterior, soleus, biceps femoris and rectus femoris muscles were recorded by IVM $\mathrm{Ag}-\mathrm{AgCl}$ electrodes attached $3 \mathrm{~cm}$ apart over the muscle bellies. With the patient in a supine position, the flexor responses were stimulated by the active peroneal and neutral lower quadriceps electrode as used for the FES during gait. Stimulation trains of $0.2 \mathrm{~ms}$ monophasic constant current pulses with frequencies of $20 \mathrm{~Hz}, 50 \mathrm{~Hz}$ and $100 \mathrm{~Hz}$ lasted $1 \mathrm{~s}$. The amplitudes were adjusted from 30 to $100 \mathrm{~mA}$ for a flexor response that would be functional during gait. Ten consecutive responses were averaged for each patient. Peak amplitudes and areas of the rectified EMG signals were assessed in a 70-500 ms sweep interval after the stimulation train. Area and amplitude ratios between the tibialis anterior and soleus and between the rectus and biceps femoris muscles were calculated.

The response itself was measured in the sagittal plane during the stimulation whilst standing. Reflective markers were attached to the hip, knee and ankle joint axes, heel and toes. A Selspot II 3D motion analysis system (Selspot AB, Möludal, Sweden) with two cameras was employed to record the stimulated flexor response in the lower limb at different frequencies. The patients themselves turned on the stimulation for $2 \mathrm{~s}$ by a manual switch, similarly during the stimulated gait. The original ALT-2 stimulator with $20 \mathrm{~Hz}$ and the modified one with $60 \mathrm{~Hz}$ were used consecutively.

\section{Results}

All four patients tolerated the modified training programme well, with immediate standing. There were no cardiovascular or orthopaedic problems. As is presented in Figure 1, they stood from 1.5 to $10 \mathrm{~min}$ in the standing-frame during the first session. They reached 25-40 min of standing in 10-17 days and the next day the FES-walking programme was initiated. The drop in the standing time during the preparatory period in the second patient (T4 lesion) in Figure 1 was due to a high fever for 2 days, which delayed the beginning of FES walking. One day drops in the standing duration in the third and fourth patient were caused by a switch from

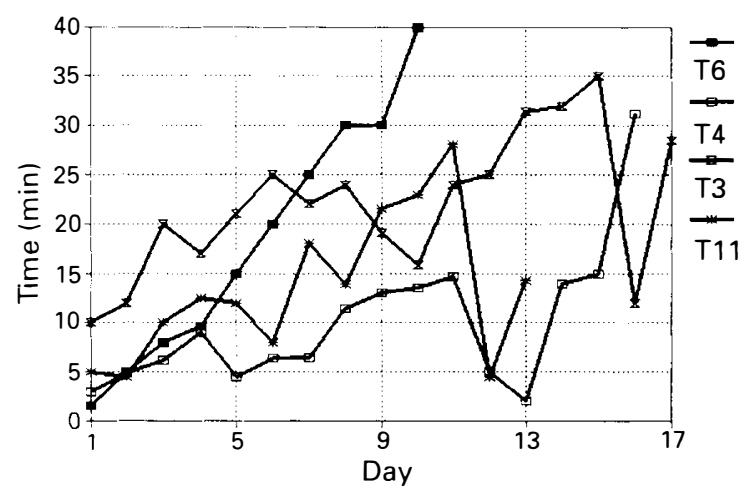

Figure 1 Standing of the four paraplegic patients by FES before the stimulated gait 
the standing frame to rolling walker before the start of FES gait.

The first patient ( $\mathrm{T} 6$ level lesion) started walking for 30 min during both sessions on the first day (Figure 2), reached $45 \mathrm{~min}$ in 5 days and over $1 \mathrm{~h}$ in 10 days. The second patient (T4 level lession) started walking for $3 \mathrm{~min}$ and managed $30 \mathrm{~min}$ in 8 days. The third patient (T3 level lesion) started walking with $12 \mathrm{~min}$, dropped to $4 \mathrm{~min}$ the next day and reached $20 \mathrm{~min}$ in 8 days. The fourth patient (T11 level lesion) started with $3 \mathrm{~min}$ and reached $30 \mathrm{~min}$ in 10 days.

The patients walked very slowly at the beginning, covering only short distances. Their walking speed gradually increased and reached a sustained gait after several weeks. Only the first patient (T6 level lesion) has been able to cover long distances and walk for $2 \mathrm{~h}$ so far. Presently he also walks with crutches and climbs stairs. The others perform FES standing and gait on shorter distances and for exercise purposes.

Vertical ground reaction forces during gait showed rather symmetrical patterns, eg mean right and left stance time ratio reached 0.94 with the corresponding swing time ratio of 0.86 . Trajectories of the force point of action on the soles revealed walking on matatarsals and toes in two patients (T4, T3), while they moved from the heel/mid-foot to toes in the other two (T6, T11). The forces of the fastest (T6) and the slowest patient (T4) during gait with the rolling walker are given in Figure 3. Velocity, cadence, stride length and arm support normalised by body weight are set out in Table I for all patients. Besides the parameters of gait with the rolling walker the gait with crutches and climbing stairs is also presented for the first patient.

The second patient, whose lower limbs had been exceedingly cold before the FES-programme, benefited from a better thermoregulation. He could abandon wool underwear and heating in bed during the summer. In the fourth patient dysaesthesias at the level of lesion were diminished. In one patient, urinary tract infection requiring antibiotic treatment occurred only once, over a period of several months.

With diminution of the flexor response after several months of the stimulation a higher stimulation frequency was applied. Amplitudes of the stimulation, adjusted for the functional flexor response, were on

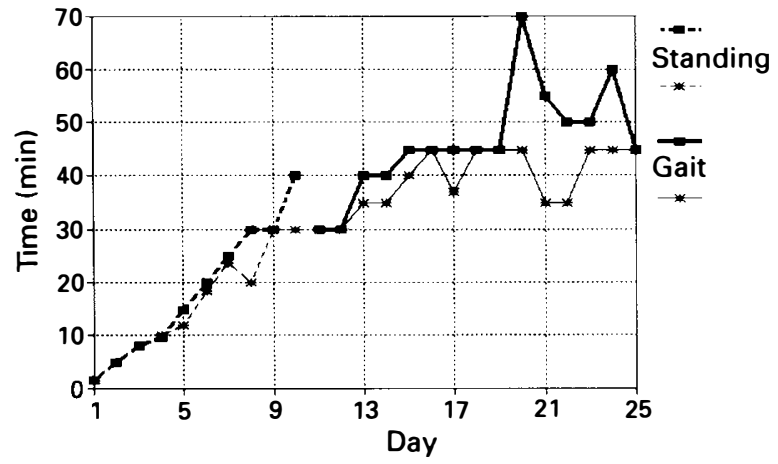

Figure 2 FES standing and gait of the T6 paraplegic patient twice a day during the first 25 days
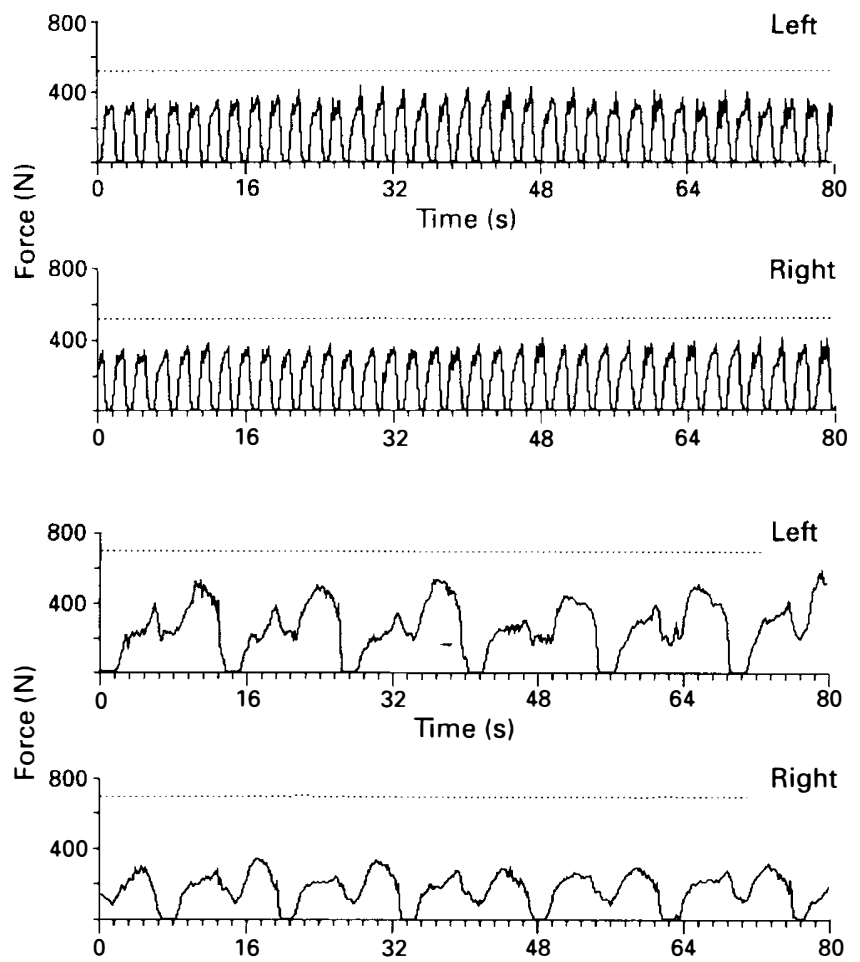

Figure 3 Vertical forces during electrically stimulated gait in 80 s trials of the fastest (T6 lesion) and the slowest paraplegic patient (T4 lesion). Dotted lines represent body weight

Table 1 Parameters of gait

\begin{tabular}{|c|c|c|c|c|}
\hline Patient & $\begin{array}{l}\text { Velocity } \\
\left(\mathrm{mmin}^{-1}\right)\end{array}$ & $\begin{array}{c}\text { Cadence } \\
\left(\text { step } \text { min }^{-1}\right)\end{array}$ & $\begin{array}{l}\text { Stride } \\
(m)\end{array}$ & $\begin{array}{c}\text { Support } \\
(\%)\end{array}$ \\
\hline \multirow[t]{3}{*}{ T6 } & 24.2 & 50 & 0.97 & 42 \\
\hline & $5.8^{\mathrm{a}}$ & $14^{\mathrm{a}}$ & $0.83^{a}$ & $43^{a}$ \\
\hline & $5.4^{b}$ & $23^{b}$ & $0.46^{\mathrm{b}}$ & $49^{b}$ \\
\hline $\mathrm{T} 4$ & 2.7 & 8 & 0.75 & 53 \\
\hline $\mathrm{T} 3$ & 3.1 & 11 & 0.60 & 65 \\
\hline T11 & 4.4 & 10 & 0.90 & 67 \\
\hline
\end{tabular}

a Walking with crutches

bClimbing stairs $(18 \times 28 \mathrm{~cm})$

average $39 \%$ lower at $50 \mathrm{~Hz}$ and $55 \%$ lower at $100 \mathrm{~Hz}$ than at the stimulation frequency of $20 \mathrm{~Hz}$. The effects of the $20 \mathrm{~Hz}$ and $50 \mathrm{~Hz}$ frequencies on the flexor response are illustrated in Figure 4. At a frequency of $50 \mathrm{~Hz}$, the activity of the tibialis anterior muscle was higher whereas activity of the soleus decreased. EMG response of the biceps femoris muscle, merely present at $20 \mathrm{~Hz}$, showed a sustained strong reflex mediated activation at $50 \mathrm{~Hz}$ at the same time. The rectus femoris muscle remained rather quiescent in both cases.

Mean area and amplitude ratios of the flexion response of all patients are presented in Figure 5 for $20 \mathrm{~Hz}, 50 \mathrm{~Hz}$ and $100 \mathrm{~Hz}$. The area ratio of the tibialis anterior and soleus muscles was 3.44 times higher at $50 \mathrm{~Hz}$ and 2.11 times higher at $100 \mathrm{~Hz}$ than at $20 \mathrm{~Hz}$. The corresponding amplitude ratio was 2.29 times 

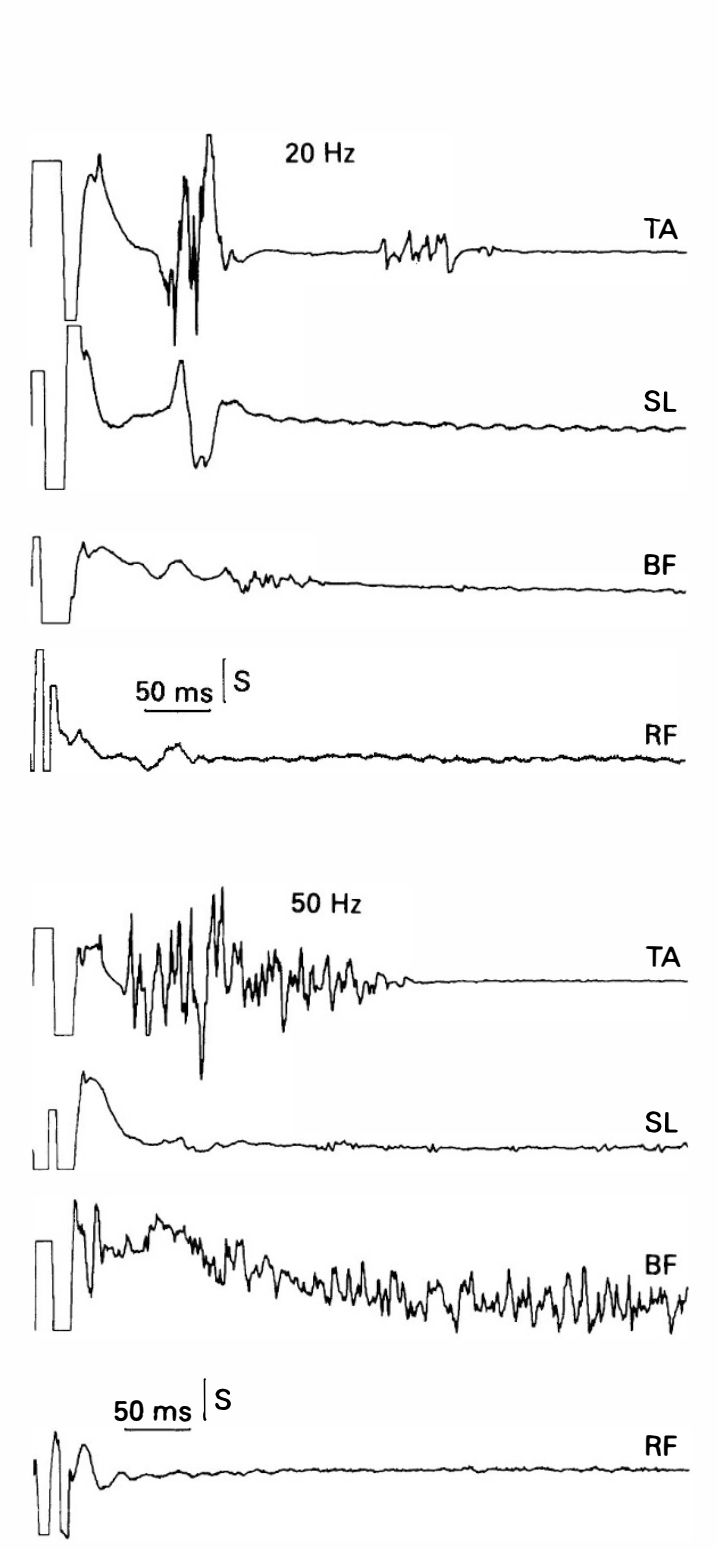

Figure 4 EMG response to stimulation frequency of $20 \mathrm{~Hz}$ and $50 \mathrm{~Hz}$ in the paraplegic patient with T6 lesion. Sensitivity (S) represents $100 \mathrm{uV}$ for the tibialis anterior (TA), $50 \mathrm{uV}$ for soleus (SL) and $20 \mathrm{uV}$ for biceps femoris (BF) and rectus femoris (RF) muscles

higher at $50 \mathrm{~Hz}$ and 2.57 times higher at $100 \mathrm{~Hz}$. With a minor dorsal, moderate or even strong plantar flexion at $20 \mathrm{~Hz}$ and a supine position, the ankle joint had strong dorsal flexion at $50 \mathrm{~Hz}$ and somewhat weaker at $100 \mathrm{~Hz}$. EMG ratios between the rectus and biceps femoris muscles showed a decrease with the increased frequency: area ratio $24 \%$ and $57 \%$ at $50 \mathrm{~Hz}$ and $100 \mathrm{~Hz}$, amplitude ratio $7 \%$ and $20 \%$ at $50 \mathrm{~Hz}$ and $100 \mathrm{~Hz}$, when compared with $20 \mathrm{~Hz}$. However the flexion response was not diminished with the higher frequencies.

The Selspot II stick-figures of the paraplegic patient with the T4 lesion in Figure 6 display better flexions of all three lower limb joints in the sagittal plane at $60 \mathrm{~Hz}$ stimulation when compared to that at $20 \mathrm{~Hz}$. Similar responses were recorded in all four patients.

Mean hip and knee flexions in the sagittal plane were

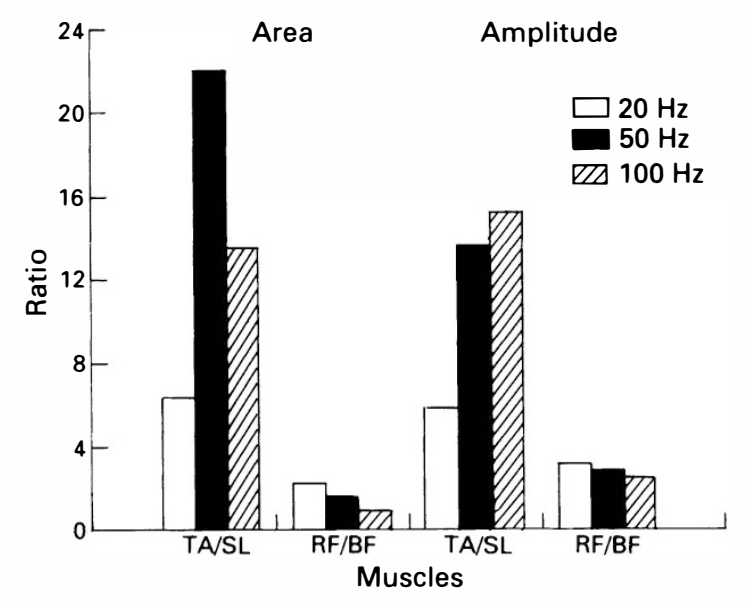

Figure 5 Mean ratios of the area and peak amplitude between tibialis anterior/soleus (TA/SL) and rectus femoris/ biceps femoris $(\mathrm{RF} / \mathrm{BF})$ rectified EMG signals of the flexion response
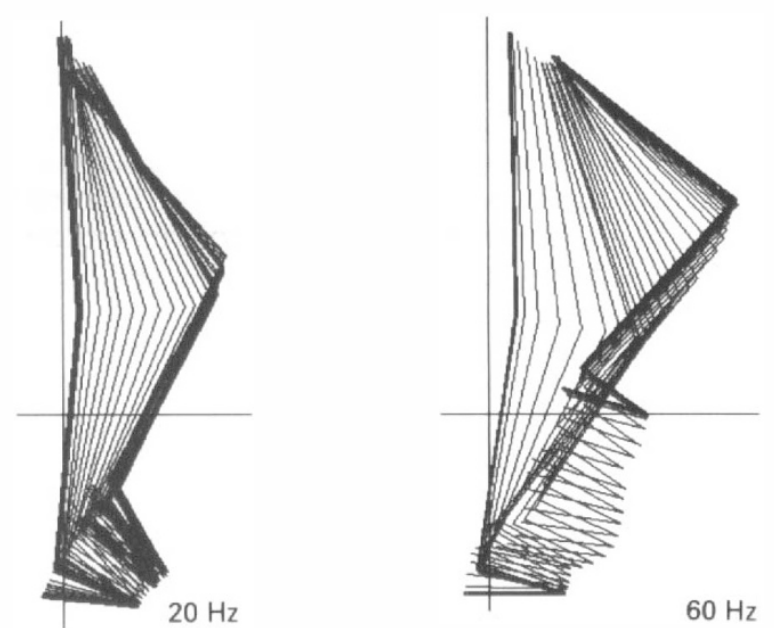

Figure 6 Effect of the $20 \mathrm{~Hz}$ and $60 \mathrm{~Hz}$ stimulation frequency on the flexor response of the paraplegic patient with T4 lesion

Table 2 Flexion response to $20 \mathrm{~Hz}$ and $60 \mathrm{~Hz}$ stimulation (negative sign indicates plantar flexion)

\begin{tabular}{lccccccc}
\hline \multirow{2}{*}{ Patient } & \multicolumn{2}{c}{$20 \mathrm{~Hz}$ stimulation } & & \multicolumn{3}{c}{$60 \mathrm{~Hz}$ stimulation } \\
\cline { 2 - 3 } \cline { 6 - 8 } & Hip & Knee & Ankle & & Hip & Knee & Ankle \\
\hline T6 & $52^{\circ}$ & $63^{\circ}$ & $-27^{\circ}$ & & $66^{\circ}$ & $97^{\circ}$ & $25^{\circ}$ \\
T4 & $30^{\circ}$ & $62^{\circ}$ & $-18^{\circ}$ & & $50^{\circ}$ & $93^{\circ}$ & $26^{\circ}$ \\
T3 & $47^{\circ}$ & $75^{\circ}$ & $-11^{\circ}$ & & $58^{\circ}$ & $90^{\circ}$ & $3^{\circ}$ \\
T11 & $39^{\circ}$ & $61^{\circ}$ & $-31^{\circ}$ & & $42^{\circ}$ & $84^{\circ}$ & $-0^{\circ}$ \\
Mean & $42^{\circ}$ & $65^{\circ}$ & $-22^{\circ}$ & & $54^{\circ}$ & $91^{\circ}$ & $14^{\circ}$ \\
\hline
\end{tabular}

$29 \%$ and $40 \%$ higher at $60 \mathrm{~Hz}$ stimulation when compared to that at $20 \mathrm{~Hz}$. At $20 \mathrm{~Hz}$ stimulation the ankle moved moderately to strongly into plantar flexion in all of the patients (mean angle $-22^{\circ}$ ). At the 
$60 \mathrm{~Hz}$ frequency and the corresponding mean angle of $14^{\circ}$, the ankle responded with the neutral position or slight dorsal flexion in two patients and with strong dorsal flexion in the other two. The improved flexion response has remained stable up to the time of this report, 6-8 months after the increase of stimulation frequency.

\section{Discussion}

It was found that intermittent stimulation of the quadriceps muscles at isotonic conditions in a lying patient might have lasted several months before sufficient knee joint torques of around $50 \mathrm{Nm}$ for the beginning of standing and walking were achieved. ${ }^{1}$ The average re-strengthening time was found to be approximately 1 month, but there was considerable variability. ${ }^{2}$ In incomplete spinal cord injury patients, strengthening of the quadriceps muscles by the stimulation and progressive resisted exercise programme with weights at the ankle lasted 6 months and was completed by all subjects. ${ }^{3}$

The alternative approach in chronic non-ambulatory hemiparetic patients with immediate walking by FES, which provided their independent gait in $2-3$ weeks ${ }^{4,5}$ excluded any previous strengthening programme. With sometimes much better preserved lower limb muscles due to spasticity and complete support of the upper limbs in paraplegic patients, immediate standing without previous conditioning was found to be rational and challenged both the research and the patient part of the team.

Standing with the help of electrical stimulation itself appeared to be a much more efficient muscle strengthening exercise than did cyclic conditioning with the patient lying down. During the latter, the patients had not been participating actively for obvious reasons; sometimes they were reading or even sleeping. By stimulated standing however, the quadriceps muscles were strengthened isometrically with active participation of the patient, including the remaining trunk and the upper extremity muscles, postural reflexes and acquaintance with the new body position.

These results correlated with a feeling of security and ability in the patient. Its importance was illustrated during the switch from standing in the frame to standing with the rolling walker in the last two patients. The ability of uninterrupted standing was reduced to $34 \%$ in the third patient and even to $14 \%$ in the fourth one during the first day with the walker. The standing time had already increased from $14 \%$ to $36 \%$ during the afternoon session of the same day in the fourth patient. During the second day with the walker it returned to $80 \%$ and $50 \%$ respectively in both patients and the next day they started walking.

Only the first patient walked with the slow speed of a healthy person. The others were able to cover up to $4 \frac{1}{2} \mathrm{~m}$ per min. With a comparable stride length up to almost $1 \mathrm{~m}$ their extremely slow gait velocity was a consequence of a very low cadence. They themselves controlled their stepping by the manual switches and spent rather a long time between the steps. They were correcting their foot contact, poor hip extension and were looking down for compensation of proprioception, which additionally reduced their hip extension. Therefore they stood in a lean-forward position and had to support themselves more with the arms. There was no direct relation between walking on fore-foot and the forward lean of the trunk, but a good one with the level of lesion. The force trajectories stayed on the fore-foot area in the patients with the T3 and T4 lesions, which might be explained by the increased muscle tone of the triceps surae muscles in these patients.

Habituation of the flexion reflex with prolonged electrical stimulation had been reported in spinal cord injury patients both in basic ${ }^{11,12}$ and clinical reports. ${ }^{6,7}$ To overcome the problem of habituation, the peroneal nerve was conditioned by high intensity stimulation. Five $5 \mathrm{~ms}$ pulses at $1 \mathrm{~Hz}$ were introduced after 20th and 40 th response to a typical $25 \mathrm{~Hz}, 0.3 \mathrm{~ms}$ stimulation. ${ }^{8}$ The high intensity conditioning could restore the habituated response to about its original level. Further, multiplexing of the conventional $25 \mathrm{~Hz}, 0.3 \mathrm{~ms}$ stimulation between the peroneal and saphenous nerves reduced the effect of habituation in some subjects. ${ }^{9}$ In the same study, latency between the onset of stimulation and the onset of hip movement, important for control aspects of FES-assisted gait, was investigated with stimulation of $25 \mathrm{~Hz}, 50 \mathrm{~Hz}$ and $100 \mathrm{~Hz}$. An overall significant decrease in the latency by increasing the frequency was reported.

Latency of the response did not represent a problem in the patients of this study. Diminished flexion response, however, required higher stimulation amplitudes at $20 \mathrm{~Hz}$ and $0.25 \mathrm{~ms}$ pulses after several months. As the patients did not use additional ankle-foot orthoses, direct stimulation of the ankle dorsal flexion was combined with a flexion withdrawal reflex by the same electrodes at the peroneal nerve. A higher amplitude increased the hip and knee flexion, but the peroneal electrode also irradiated the stimulation to the tibial nerve in the vicinity. Due to the stronger triceps surae muscles plantar flexion prevailed over dorsal flexion at the ankle, thus impeding the functional flexion response by a diminished foot clearance. The small $1 \mathrm{~cm}$ peroneal electrodes for more selective stimulation at lower intensity provided only a temporary solution.

EMG analysis showed an increased ratio of peak amplitudes between the tibialis anterior and soleus muscles in favour of dorsal flexion with the increased stimulation frequency. However, the sustained activation, represented by the area ratio between the tibialis anterior and soleus muscles, was higher at $50 \mathrm{~Hz}$ and partly decreased at $100 \mathrm{~Hz}$. At the $100 \mathrm{~Hz}$ stimulation, the lower stimulation intensity was still sufficient for a reflex activation of both muscles. The decrease at $100 \mathrm{~Hz}$ could be explained by a proportionally increased fatigue at this stimulation frequency in the tibialis anterior muscle, which was, in contrast to the triceps surae, additionally directly stimulated. 
With the improved EMG image, the measured angles displayed a better flexion pattern at the higher frequency. Besides the improved hip and knee flexions, ankle movement was shifted into dorsal flexion. The mean ankle angle of $22^{\circ}$ of plantar flexion (range $11^{\circ}-31^{\circ}$ ) in these patients indicated a spread of direct stimulation to the tibial nerve by the electrodes over the nearby peroneal nerve and the high amplitude required to elicit the flexion response at $20 \mathrm{~Hz}$. With the better reflex mediated hip and knee flexions with the lower amplitude at $60 \mathrm{~Hz}$, two patients showed a neutral to slight, and the other two strong dorsal flexion responses. Direct cross-stimulation of the tibial nerve was not observed at this frequency. Improvement of ankle movement at $60 \mathrm{~Hz}$ was uniform and exceeded $100 \%$ in all of the patients when compared to $20 \mathrm{~Hz}$. Therefore not only a reflex mediated, but also a direct stimulation of the ankle dorsal flexors appeared to be important for the stimulated complex lower limb movement.

\section{Conclusion}

Instead of the tedious intermittent isotonic or progressively loaded muscle strengthening active, electrically stimulated standing was found to be a more efficient muscle strengthening programme in our spinal cord injury patients. By daily standing with the help of electrical stimulation the preparatory period was substituted by active exercise which acquainted them with the new body position and enabled them to start walking in 10 to 17 days.

With $500-1000 \mathrm{~Hz}^{10}$ or $2000 \mathrm{~Hz}$ stimulation of the flexion reflex in neurophysiological studies, ${ }^{11,12}$ FES frequencies around $20 \mathrm{~Hz}$ from clinical reports were well justified for the antigravity muscles in order to reduce fatigue, while the reason for their application in the flexion response was not so obvious. The present work attested that an increased frequency rendered a better flexor response also after a long term application.

As the reflex response was combined with direct stimulation of ankle dorsal flexion, the frequency of $60 \mathrm{~Hz}$ was selected as an optimum. It also offered a viable solution for small portable stimulators. For this reason and from the aspect of fatigue, the frequency for the quadriceps muscle was reduced to $16 \mathrm{~Hz}$, which still provided a sustained, strong enough contraction.

\section{Acknowledgements}

The authors are indebted to Ms Daniela Lücke for her help with the patients and preparation of the manuscript. The study was supported by a grant (91.050.1) from the Wilhelm Sander, Stiftung.

\section{References}

1 Kralj A, Bajd T. Functional Electrical Stimulation: Standing and Walking After Spinal Cord Injury. CRC Press Inc: Boca Raton, Florida, 1989.

2 Cybulski GR, Penn RD, Jaeger RJ. Lower extremity functional neuromuscular stimulation in cases of spinal cord injury. Neurosurgery 1984; 15: 132-146.

3 Granat MH, Ferguson ACB, Andrews BJ, Delargy M. The role of functional electrical stimulation in the rehabilitation of patients with incomplete spinal cord injury-observed benefits during gait studies. Paraplegia 1993; 31: 207-215.

4 Maležič $\mathrm{M}$ et al. Evaluation of gait with multichannel electrical stimulation. Orthopedics 1987; 10: 769-772.

5 Bogataj U et al. Restoration of gait during two to three weeks of therapy with multichannel electrical stimulation. Phys Ther 1989; 69: 319-327.

6 Jaeger RJ, Kralj A. Studies in functional electrical stimulation for standing and forward progression. Proceedings of Sixth Annual Conference on Rehabilitation Engineering, 1983; San Diego, California, RESNA, Bethesda, Maryland, pp 75-77.

7 Andrews BJ, Baxendale RH, Granat RH, Nicol DJ. Flexion withdrawal reflexes in spinal cord injured man. J Physiol 1990; 429: 50P.

8 Nicol DJ, Granat MH, Baxendale RH, Andrews BJ. Dishabituation of the flexion reflex in spinal man. J Physiol 1991; 429: $17 \mathrm{P}$

9 Granat $\mathrm{MH}$ et al. Improving limb flexion in FES gait using the flexion withdrawal response for the spinal cord injured person. J Biomed Eng 1993; 15: 51-56.

10 Hagbarth KE. Spinal withdrawal reflexes in human lower limbs. J Neurol Neurosurg Psychiatry 1960; 23: 222-227.

11 Dimitrijević MR, Nathan PW. Studies of spasticity in man: 4. changes in flexion reflex with repetitive cutaneous stimulation in spinal man. Brain 1970; 93: 743-768.

12 Dimitrijević MR, Nathan PW. Studies of spasticity in man: 5. dishabituation of the flexion reflex in spinal man. Brain 1971; 94: 77-90. 